Centro de Referência e Treinamento em DST/AIDSSão Paulo, Programa Estadual de DST/aids-São Paulo, Coordenadoria de Controle de Doenças, Secretaria de Estado da Saúde de São Paulo

Contato:

Imatida@gmail.com

\section{Eliminação da transmissão vertical do HIV e da sífilis no Estado de São Paulo}

\section{Eliminating vertical transmission of HIV and syphilis in São Paulo State, Southeastern Brazil}

A transmissão vertical do HIV e da sífilis se mantém como um grande desafio para a saúde pública, demandando novas estratégias de vigilância epidemiológica integrada, devido ao complexo processo infeccioso e ao comportamento da população.

A eliminação da transmissão vertical do HIV e da sífilis pode ser alcançada caso esses agravos sejam prevenidos e/ou diagnosticados e tratados durante o período de prénatal. A Organização Panamericana da Saúde (Opas) define a eliminação da transmissão vertical do HIV quando são alcançadas taxas de incidência menores de dois casos de HIV para cada 100 mães infectadas, e, para a sífilis congênita, considera que a doença é eliminada quando existe a ocorrência de menos de um caso para cada 1.000 nascidos vivos. Existe ainda a possibilidade de prevenir a transmissão do HIV no momento do parto, o que não é possível para a sífilis. A taxa de infecção da transmissão vertical do HIV pode ser reduzida a níveis inferiores a $2 \%$ e a sífilis congênita pode ser evitada se a mãe for diagnosticada e tratada adequadamente durante o pré-natal.

A comparação das ações para o controle das duas doenças mostra importantes diferenças. No Brasil, embora essas intervenções estejam preconizadas e teoricamente recomendadas para toda a população de gestantes infectadas e seus filhos, as dificuldades da rede em prover acolhimento, aconselhamento e diagnóstico laboratorial da infecção, bem como a cobertura insuficiente de mulheres testadas no pré-natal, principalmente nas populações mais vulneráveis e a variável qualidade do pré-natal, ainda aquém do desejável, resultam em uma utilização desigual e ainda bastante precária em algumas regiões.

O Estado de São Paulo possui 40 milhões de habitantes em 645 municípios e realiza $600 \mathrm{mil}$ partos/ano. A Secretaria de Estado da Saúde e áreas afins (Atenção Básica, Mulher, Criança, Laboratório, Vigilâncias Epidemiológica e Sanitária, Programa de DST/aids, Câmara Técnica de DST/aids), Conselho dos Secretários Municipais de Saúde e Agência Nacional de Saúde
Suplementar elaboraram o "Plano de Eliminação da Transmissão Vertical do HIV e da Sífilis até 2015".

A taxa de infecção da transmissão vertical do HIV no Estado de São Paulo diminuiu de 9,4\% em 2000 para 2,7\% em 2006 (Figura 1). À semelhança de grandes centros internacionais, a notificação de casos de sífilis na gestação aumentou (152 casos em 2005 para 1.709 em 2009), assim como o número de municípios notificantes (Figura 2), e o tratamento dos parceiros sexuais ainda é um grande desafio, pois os homens, de maneira geral, pouco comparecem para atividades preventivas nos serviços básicos de saúde (Tabela).

Diante dessa desigualdade de expressão epidemiológica e de priorização do ponto de vista político dessas duas condições, é necessário e urgente antecipar o enfoque da sífilis na gestante, um agravo que tem diagnóstico e tratamento disponíveis, mas que vem se mostrando um desafio para a Saúde Pública.

\section{PLANO DE ELIMINAÇÃO DA TRANSMISSÃO VERTICAL DO HIV E DA SÍFILIS NO ESTADO DE SÃO PAULO}

O Programa Estadual de DST/aids de São Paulo (PEDST/aids-SP) vem apoiando a ampliação e manutenção das ações de prevenção da transmissão vertical do HIV e sífilis na rede de serviços (atenção primária, unidades de referência, maternidades) gerenciada diretamente pelo estado e municípios. Atualmente, todos os insumos utilizados para a prevenção da transmissão vertical do HIV e sífilis são financiados pelo Governo.

Em 2009, o PEDST/aids-SP lançou o "Plano de Eliminação da Transmissão Vertical do HIV e da Sífilis”, em consonância com as propostas do Ministério da Saúde e com a Opas, com metas escalonadas e regionalizadas de redução da transmissão vertical no estado. Atualmente, constituem importantes desafios: o aumento na cobertura de testagem para HIV e sífilis no pré-natal; o tratamento da sífilis (gestantes e seus parceiros) no pré- 


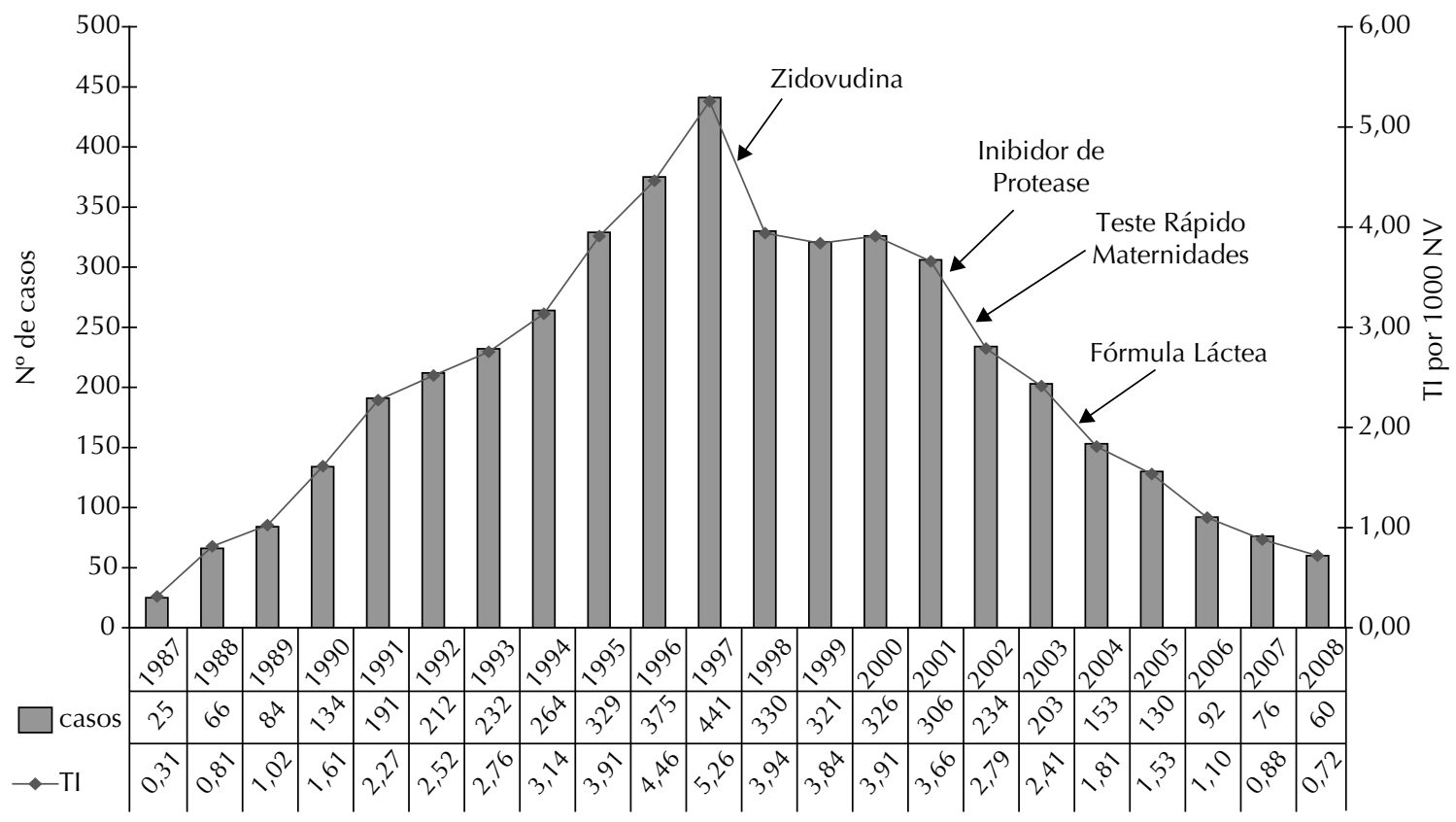

TI: Taxa de injeção

Fonte: SINAN - Vigilância Epidemiológica - Programa Estadual DST/Aids - SP. Dados preliminares até 30/06/2009 sujeitos à revisão mensal.

Figura 1. Casos de aids por transmissão vertical, segundo ano de diagnóstico e intervenções preventivas. Estado de São Paulo, 1987-2008.

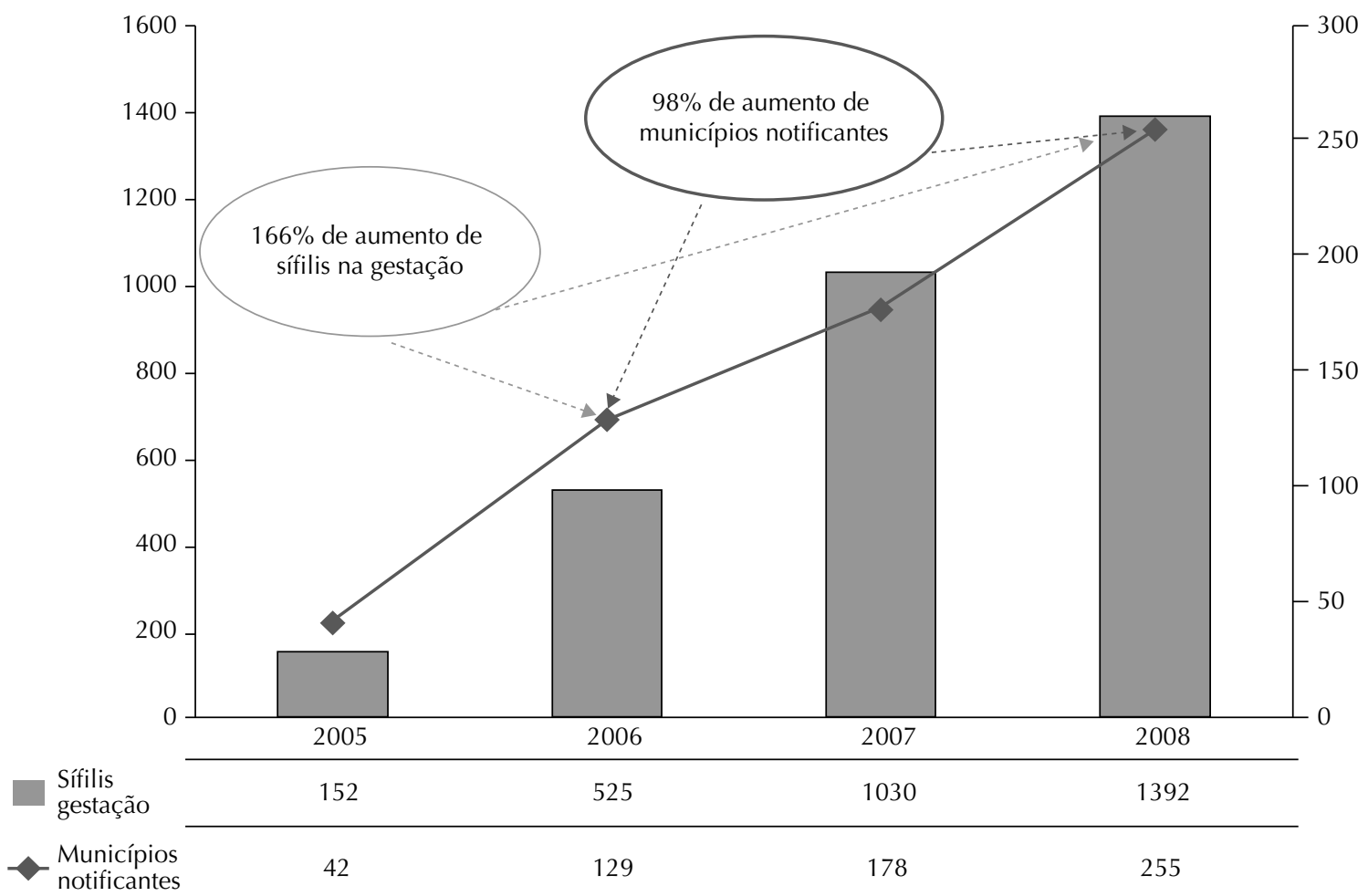

Fonte: SINAN - Vigilância Epidemiológica - Programa Estadual DST/Aids - SP (VE - PEDST/Aids-SP), dados preliminares até 18/03/09, sujeitos a revisão mensal.

Figura 2. Casos notificados de sífilis na gestação e número de municípios com casos residentes no segundo ano. Estado de São Paulo, 2005-2008. 
Tabela. Casos de sífilis congênita, segundo o tratamento do parceiro e ano de notificação.

\begin{tabular}{lcccc}
\hline \multirow{2}{*}{$\begin{array}{l}\text { Tratamento do } \\
\text { Parceiro }\end{array}$} & \multicolumn{2}{c}{ Ano de Notificação - São Paulo } \\
& $\mathrm{n}$ & $\%$ & $\mathrm{n}$ & $\%$ \\
\hline Sim & 99 & 14,8 & 74 & 11,9 \\
Não & 291 & 43,5 & 457 & 73,2 \\
Ignorado ou Branco & 279 & 41,7 & 93 & 14,9 \\
\hline Total & 1.017 & 100,0 & 1.462 & 100,0 \\
\hline
\end{tabular}

natal; e a ampliação do número de maternidades que efetivamente realizem todas as medidas de profilaxia da transmissão vertical do HIV e sífilis durante o parto e puerpério. Municípios nos quais houve sensibilização e empenho para se reduzir essa via de transmissão para o HIV apresentam números semelhantes aos dos países desenvolvidos.

A ocorrência desses agravos entre crianças é um evento sentinela da qualidade da atenção materno-infantil e/ou de contextos de vulnerabilidades às DST/aids (morador de rua, uso de drogas, pessoas vivendo em regime prisional e seus familiares, migração, exclusão social e outros) que exigem medidas de intervenção de acordo com a realidade e necessidade local.

Como elemento fundamental de enfrentamento desses dois agravos, as ações preventivas e profiláticas precisam ser reforçadas na assistência ao pré-natal e ao parto, com a triagem laboratorial de todas as gestantes no pré-natal (na primeira consulta e no início do terceiro trimestre) e no parto, com o tratamento oportuno e adequado também ao parceiro sexual.

É de fundamental importância o envolvimento das áreas técnicas da atenção básica, da mulher, da criança e DST/ aids, além da sociedade civil em todos os níveis de atuação, e a inclusão do tema na agenda dos gestores, pois os agravos têm diagnóstico e tratamento disponíveis.

O PEDST/aids-SP propõe que seja investigado todo caso diagnosticado e notificado de sífilis congênita, assim como todo caso diagnosticado e notificado de aids em crianças, a fim de que sejam detectadas todas as oportunidades perdidas que essa gestante, seu parceiro sexual e essa criança sofreram. A identificação dessas oportunidades perdidas deverá orientar o planejamento, monitoramento e avaliação das ações para o controle desses agravos.

Esse plano estratégico identifica os problemas e suas conseqüentes metas, suas ações e os executores responsáveis. Com relação à sífilis, algumas ações são:

1) apresentação do Plano a 600 gestores municipais, com premiação de experiências municipais;
2) pactuação junto à bipartite estadual (representantes da Secretaria de Estado da Saúde e também das secretarias municipais), com controle da execução do teste treponêmico nas maternidades e notificação negativa da sífilis congênita;

3) publicação de notas técnicas: algoritmo de diagnóstico da sífilis com a inclusão do teste treponêmico quando da identificação do teste não treponêmico reagente; e consulta do parceiro sexual durante o pré-natal;

4) divulgação de iniciativas municipais;

5) divulgação de cartazes relativos à responsabilidade do homem na transmissão vertical da sífilis;

6) divulgação do Plano aos médicos cadastrados no Conselho Regional de Medicina;

7) constituição do Grupo de Estudo da Transmissão Vertical do HIV e da Sífilis;

8) realização de eventos regionais com a participação de 4.000 gestores e técnicos.

Apesar de a sífilis congênita ser um agravo com clínica, diagnóstico e tratamento conhecidos já há muito tempo, várias situações polêmicas e de estruturação da rede de saúde dificultam sua eliminação. Esse Plano aborda as situações detectadas e aposta nas estratégias preventivas.

A sífilis não tratada durante a gestação resulta em uma considerável proporção de mortes fetais e neonatais precoces, agravando o quadro epidemiológico da sífilis congênita. Portanto, há necessidade urgente da qualificação da atenção pré-natal com garantia dos insumos necessários específicos para a sífilis, momento crucial para se atingir a meta de eliminação do agravo.

A expansão da epidemia do HIV/aids na população feminina acarretou o aumento dos casos de aids em crianças adquiridos pela transmissão vertical do HIV. Nos países desenvolvidos, a ampla implementação de intervenções para a redução dessa modalidade de transmissão do HIV, principalmente a administração de anti-retrovirais, a cesariana eletiva e a substituição do aleitamento materno resultaram na redução significativa da incidência de casos de aids em crianças.

Ações de controle com cobertura e qualidade consideradas satisfatórias promovem impactos substanciais na redução de novos casos de crianças infectadas. Apesar das desigualdades ainda existentes em todo o mundo, esses resultados epidemiológicos e operacionais também são observados em alguns países em desenvolvimento, incluindo o Brasil. O País vem se

\footnotetext{
a Boletim Epidemiológico. São Paulo: Secretaria de Estado da Saúde, Centro de Referência e Tratamento DST/AIDS. Ano 27, №. 1, dez 2010.
} 
destacando no cenário internacional pelo compromisso e responsabilidade com a implementação de ações para o enfrentamento da infecção por HIV e de outras DST.

O Estado de São Paulo foi responsável por 38,3\% (176.946 casos) do total de casos notificados de aids no País, entre 1980 e junho de 2009. São Paulo representou, nesse período, $36,5 \%$ (4.744) do total de casos de aids em crianças menores de 13 anos de idade no Brasil. Esse Estado foi o pioneiro no País ao criar, em 1983, o PEDST/aids, buscando implementar as ações de controle da infecção por HIV no geral, incluindo a transmissão vertical do HIV. ${ }^{\text {a }}$

O Estado foi também pioneiro no País na adoção de medidas preventivas da transmissão vertical do HIV, já a partir de meados dos anos 1990, com base nas primeiras evidências internacionais geradas pelo estudo ACTG076 em 1994, de redução de 67,5\% da transmissão com a instituição da zidovudina no prénatal, parto e pós-parto. Naquele mesmo ano iniciou a implantação do protocolo no estado, enquanto nacionalmente eram definidas as diretrizes para prevenção dessa modalidade de transmissão.

Reconhece-se que a eliminação da transmissão vertical do HIV é uma possibilidade concreta no Brasil com base nas políticas nacionais e na organização dos serviços de saúde, bem como nas evidências científicas disponibilizadas, se obedecidas todas as recomendações preconizadas. Apesar de no Brasil haver disponibilidade dos insumos necessários à interrupção da transmissão vertical do HIV nos serviços públicos de saúde (teste anti-HIV, medicamentos anti-retrovirais, inibidor da lactação e fórmula láctea infantil), apenas 52\% das gestantes brasileiras receberam cobertura efetiva das ações de prevenção da transmissão vertical no pré-natal; no estado de São Paulo essa cobertura foi de $82 \%$.

Para a redução da incidência da aids em menores de 13 anos, a rede de atenção deve ser adequada para a revisão dos fluxos de referência e contra-referência entre os serviços de pré-natal e laboratórios. Isso inclui o uso de novas tecnologias, como a aplicação do teste rápido para o diagnóstico do HIV nas maternidades, a ampliação da cobertura das ações de profilaxia da transmissão vertical do HIV em gestantes, parturientes e em crianças expostas.

Em relação às crianças expostas, a não-realização da profilaxia (ou realização por menos de seis semanas) e exposição ao leite materno foram definidas como situações de maior vulnerabilidade para a transmissão vertical, mostrando-se fatores determinantes de sua ocorrência.

Os dados atualmente observados no estado para a infecção por HIV em crianças por transmissão vertical são comparáveis aos observados em muitos países desenvolvidos, apesar de permanecerem importantes questões operacionais, mesmo com a disponibilidade de todas as condições para o seu enfrentamento.

Esse declínio, em outras proporções, também foi verificado por estudos que ressaltam o impacto das ações de controle da transmissão vertical do HIV, como o aumento do tempo de sobrevida e conseqüente melhoria da qualidade de vida de crianças que estão conseguindo avançar para a idade adulta.

Apesar de ainda haver subnotificação e mesmo pacientes que por razões não identificadas não têm acesso ao tratamento, nos últimos anos, a incidência de casos de aids em crianças por exposição vertical vem decrescendo progressivamente no Brasil. Diferentemente, a sífilis congênita apresenta manutenção de sua freqüência e até aumento significativo em algumas regiões.

No Estado de São Paulo, pretende-se ampliar o debate nos vários segmentos da sociedade envolvidos com esse enfrentamento, diminuindo o estigma em relação às DST e ao HIV/aids. Uma vez que esses agravos tornem-se mais visíveis como um problema para a população em geral e para os profissionais de saúde, isso pode contribuir para o tão almejado alcance da eliminação da transmissão vertical do HIV e da sífilis. 